\title{
Influências na alteração do regime de bloqueios atmosféricos sobre o sul do Brasil em um cenário de clima futuro
}

\author{
Influences on Atmospheric Blocking regimes on Southern Brazil on scenarios of \\ future climate change
}

\author{
Diego Pedroso ${ }^{1}$, Simone Erotildes Teleginski Ferraz ${ }^{1}$, Ernani de Lima Nascimento ${ }^{1}$, Tércio \\ Ambrizzi $^{2}$, Rosmeri Porfírio da Rocha ${ }^{2}$ \\ ${ }^{1}$ Departamento de Estatística, UFSM, Santa Maria, Brasil \\ ${ }^{2}$ Instituto Astronômico, Geofísico e de Ciências Atmosféricas, USP, São Paulo, Brasil
}

\begin{abstract}
Resumo
Bloqueios atmosféricos são fenômenos que ocorrem, especialmente, em latitudes médias, com duração que pode variar entre alguns dias, chegando a permanecer ativo por semanas. Durante a ocorrência de um bloqueio atmosférico, surge uma anomalia persistente de alta pressão, localizada ao sul do cinturão das altas subtropicais semipermanentes, impedindo que os sistemas avancem em seu curso normal. Para detectar estes eventos foi utilizado o índice proposto por Pelly e Hoskins (2003) - PH03. Neste índice é detectada a inversão do gradiente meridional de temperatura potencial na tropopausa dinâmica (altos níveis), causada pela circulação anômala persistente. A situação deve permanecer ativa por mais de três dias consecutivos e estar presente em uma área maior que $10^{\circ}$ de longitude. O índice será calculado para o período de inverno de 1986, além disso, será feita a análise dos campos meteorológicos que caracterizam as suas condições sinóticas. O bloqueio permaneceu ativo na região durante vários dias entre o final do mês de julho e a primeira quinzena de agosto. Os resultados se mostraram satisfatórios, de modo que a análise dos campos meteorológicos mostrou o estabelecimento da condição de bloqueio, mostrando a funcionalidade do indice PHO3.
\end{abstract}

Palavras-chave: Bloqueios atmosféricos. clima futuro

\begin{abstract}
Blockings are atmospheric phenomena occurring especially in midlatitudes with duration ranging from a few days, reaching remain active for weeks. During the occurrence of an atmospheric blocking, a persistent high pressure anomaly, located south of the belt of semi-permanent subtropical high, preventing the advance systems in their normal course arises. To detect these events proposed by Pelly e Hoskins (2003) - (PH03) index was used. This index detect the reversal meridional gradient of potential temperature on the dynamic tropopause (high levels) caused by persistent abnormal movement. The situation must remain active for more than three consecutive days and be present in greater than $10^{\circ}$ of longitude area. The index is calculated for the period from 1986 winter, moreover, analysis of meteorological fields that characterize its synoptic conditions is made. The blockade remained active in the region for several days between the end of July and early August. The results were satisfactory so that the field of meteorological analysis showed the property of blocking condition showing the functionality of the PHO3 index.
\end{abstract}

Keywords: Atmospheric blocking. future climate. 


\section{Introdução}

Ao longo do ano, uma extensa gama de sistemas meteorológicos atua sobre a América do Sul. O mesmo pode-se dizer do Brasil, que, devido à sua grande extensão territorial, registra diversos regimes climáticos modulados através da ocorrência desses sistemas. Uma atenção especial deve ser dada aos bloqueios atmosféricos, um fenômeno que ocorre, especialmente, em latitudes médias, com duração que pode variar entre alguns dias, chegando a permanecer ativo por semanas. Nestas latitudes é comumente observado um padrão de escoamento zonal em altos níveis, caracterizado por um fluxo dirigido de oeste para leste. Nesta faixa latitudinal, encontram-se os máximos valores de magnitude do vento zonal (u), que contribuem, de forma significativa, para o deslocamento dos distúrbios transientes para leste, segundo Peixoto e Oort (1992).

Durante a ocorrência de um bloqueio atmosférico, surge uma anomalia persistente de alta pressão, localizada ao sul do cinturão das altas subtropicais semipermanentes. Esse quadro impede que os sistemas avancem em seu curso normal, caracterizando uma situação clássica de bloqueio. Pode-se observar um anticiclone quente que se forma em latitudes mais altas do que aquelas onde se localiza a alta subtropical. Frequentemente, o mesmo encontra-se acompanhado por uma baixa fria em latitudes mais baixas, segundo Cavalcanti et al. (2009). Quando esse tipo de sistema se configura, as condições a ele associadas persistem durante dias, modificando os padrões de precipitação e temperatura em uma grande região. Desta forma, a identificação de um bloqueio tem grande importância para fins de previsão do tempo e previsões climáticas de médio prazo. O estabelecimento de uma condição de bloqueio aumenta, significativamente, o grau de acerto dos modelos, devido ao caráter persistente tanto no tempo (dias ou semanas) como no espaço (estacionário). Investigá-los e verificar de que maneira eles são representados pelos modelos torna-se crucial para aumentar sua confiabilidade.

Para o Hemisfério Norte, os primeiros estudos sobre os bloqueios datam o final da década de 1940, com o pioneiro estudo realizado por Willet (1949), no qual identifica situações em que o escoamento possui uma componente meridional mais acentuada do que a observada em média nas regiões baroclínicas. Essa componente meridional acabava por obstruir a passagem dos sistemas sinóticos na região. Já o estudo de Rex (1950) produziu o que se tornou a primeira climatologia de bloqueios. $\mathrm{O}$ autor, analisando dados de altura geopotencial em $500 \mathrm{hPa}$, identificou áreas preferenciais de ocorrência, persistência e sua variabilidade sazonal e anual de forma qualitativa. Já, para o Hemisfério Sul, esses estudos iniciaram somente a partir da década de 1980.

Existem dois tipos principais de bloqueios: o tipo "ômega" e o tipo dipolo. O bloqueio tipo "ômega" consiste do domínio de uma alta pressão em $500 \mathrm{hPa}$.
Com essa configuração, o escoamento de oeste em altos níveis contorna este padrão no formato da letra grega $\mathrm{W}$ (ômega invertido do HS), caracterizando a bifurcação do jato. Já o tipo dipolo caracteriza-se por uma baixa desprendida (cutoff low) em direção das latitudes mais baixas, encontrando-se meridionalmente em fase com um anticiclone de bloqueio nas latitudes mais altas. Quando este tipo de sistema se configura, as condições a ele associadas persistem durante dias, modificando os padrões de precipitação e temperatura em uma grande região.

\section{Dados e metodologia}

A base de dados, na qual será investigada a ocorrência dos bloqueios atmosféricos, utilizada neste estudo, faz parte do CMIP5 (Coupled Model Intercomparsion Project - Phase 5). Fazem parte desta base os mais avançados modelos climáticos dos grandes centros de pesquisa ao redor do mundo. Os produtos gerados para compor essa base serão utilizados nas projeções dos cenários futuros de mudança do clima global. Dentre os diversos modelos que fazem parte da iniciativa do CMIP5, o centro britânico MOHC (Met Office Hadley Centre) disponibilizou, através de parcerias com instituições brasileiras, a versão do modelo HadGEM2-ES (Hadley Centre Global Environment Model version 2 - Earth System), descrito conforme Collins et al. (2008).

A família de modelos HadGEM2 compreende a configuração de modelos que incorporam diferentes níveis de complexidade, mas com uma estrutura física comum. A família HadGEM2 inclui uma configuração acoplada oceano-atmosfera, com uma extensão vertical da atmosfera, de modo a incluir uma dinâmica estratosférica bem resolvida. Já a configuração do sistema terrestre inclui a dinâmica da vegetação, a biologia do oceano e a química da atmosfera. Os membros da família HadGEM2 serão usados no AR5 (Assessment Report - 5), que será o Quinto Relatório de Avaliação do IPCC, discutido nos anos de 2013/2014.

A componente da atmosfera padrão do modelo possui 38 níveis verticais, em coordenadas híbridas que se estendem até, aproximadamente, $40 \mathrm{~km}$ de altura. A resolução horizontal é de 1,25 graus de latitude por 1,875 graus de longitude, produzindo uma grade global de $192 \times 145$ pontos. Isso é equivalente a uma resolução da superfície de cerca de $208 \mathrm{~km}$ x $139 \mathrm{~km}$ no Equador, reduzindo, gradativamente, a $120 \mathrm{~km} \times 139 \mathrm{~km}$, até 55 graus de latitude. Já a componente oceânica utiliza uma grade com resolução de 1 grau de latitude-longitude. A resolução de 1 grau de latitude entre os pólos e 30 graus Norte / Sul aumenta, suavemente, até um terço de um grau no equador, dando $360 \times 216$ pontos de grade no total e 40 níveis irregularmente espaçados na vertical, com uma resolução de $10 \mathrm{~m}$ próximo à superfície.

A base de dados, descrita acima, serve como condição 
de contorno para a última versão do modelo RegCM4, que foi disponibilizada à comunidade científica em 2010. Esta nova versão está preparada para ler os dados provenientes do HadGEM2 disponíveis no período de 1949 a 2100, no GPC (Grupo de Pesquisas em Clima) da UFSM. Com isso, torna-se mais fácil a manipulação dos campos do modelo britânico, além de ser possível utilizar as saídas do RegCM4 para analisar o impacto de outras variáveis meteorológicas causadas pelos bloqueios no clima futuro.

O período compreendido entre os anos de 1949 e 2005 faz parte da série denominada Historical. Essa série foi executada utilizando dados climáticos passados, provenientes de testemunhos de gelo, série de concentração de CO de Mauna Loa, dados médios globais da NOAA, forçantes antropogênicas como gases de efeito estufa, mudança de uso da terra, aerossóis e forçantes naturais, como erupções vulcânicas e energia solar, entre outras fontes. (Jones et al. , 2011)

A partir de 2005, onde é iniciado o período de simulações futuras, as mesmas são compostas por quatro cenários, denominados $\mathrm{RCP}^{\prime} \mathrm{s}$ (Representative Concentation Pathways), compostos pelos: RCP3-PD, RCP4.5, RCP6 e RCP8.5. Como os nomes indicam, eles diferenciam-se entre si, principalmente, em relação à "forçante radiati$v^{\prime \prime}$, que considera um incremento no saldo de energia no sistema terrestre (Wm2), desencadeado por todas as emissões de gases de efeito estufa.

No cenário RCP3-PD, as emissões se dirigem a níveis de concentração muito baixos de gases de efeito estufa, sendo o cenário mais otimista chamado de "pico e declínio" (PD). O nível de forçante radiativa atinge, primeiramente, um valor de cerca de $3,1 \mathrm{Wm} 2 \mathrm{em}$ meados do século XXI, voltando a cair para 2,6 Wm2 em 2010, baseado na publicação de van VUUREN et al. (2007).

No cenário RCP4.5 (disponível no GPCUFSM), a estabilização radiativa global ocorre em torno dos 4,5 Wm2 e antes do ano de 2100. Considerado o cenário intermediário que mescla o emprego de uma variedade de tecnologias, políticas ambientais e estratégias para reduzir as emissões de gases de efeito estufa. Detalhes adicionais sobre a simulação do uso da terra e as emis- sões de carbono terrestre nesse cenário é dada por Wise et al. (2009).

O RCP6.0 é um cenário estabilizado somente após o ano de 2100, em torno de $6 \mathrm{Wm} 2$, e que não há preocupação com o emprego de uma variedade de tecnologias e estratégias para reduzir as emissões de gases de efeito estufa, com detalhes descritos em Fujino et al. (2006).

O RCP8.5 é caracterizado pelo aumento desenfreado das emissões, levando a altos níveis de concentração de gases de efeito estufa com a forçante radiativa em valores maiores que $8,5 \mathrm{Wm} 2$, detalhado em Riahi et al. (2007). A concentração de CO2 equivalente (CO2-eq), por exemplo, é calculada a partir da relação mostrada na Figura 1, baseado nos cenários brevemente descritos acima. Os valores das estimativas de concentração de CO2-eq aumentam, gradativamente, até o ponto descrito pela relação:

$$
\left[\mathrm{CO}_{2}\right]=278,0 \times \mathrm{e}^{(\text {Forçante radiativa/5,325) }}
$$

Para manipular os dados do modelo HadGEM2-ES, será utilizada a fase de pré -processamento do modelo RegCM4, que as utiliza como condição de contorno. O HadGEM2-ES, por ser um modelo de mudança climática que engloba um período muito grande, possui seu calendário escrito de tal forma que todos os meses apresentem apenas 30 dias cada e, consequentemente, os anos possuem apenas 360 .

A sequência de rotinas converte os níveis verticais, inicialmente, em coordenadas híbridas para níveis sigma (s), utilizadas pelo modelo em seu processamento. Para esta fase do estudo, somente o preparo dos dados de entrada (input), que alimentarão o código do modelo RegCM4, foi realizado. Estes campos de entrada contém dados de temperatura $(\mathrm{K})$, pressão $(\mathrm{hPa})$, vento zonal e meridional (m/s), geopotencial (mgp) e umidade específica $(\mathrm{g} / \mathrm{kg})$ em 11 níveis verticais. Uma rotina do modelo converte os níveis sigma em coordenadas isobáricas que serão utilizadas no cálculo do índice de bloqueio.

Com a base de dados pré-processada pelo RegCM4, é possível identificar se um escoamento possui ou não uma condição de bloqueio. O índice é baseado na técnica

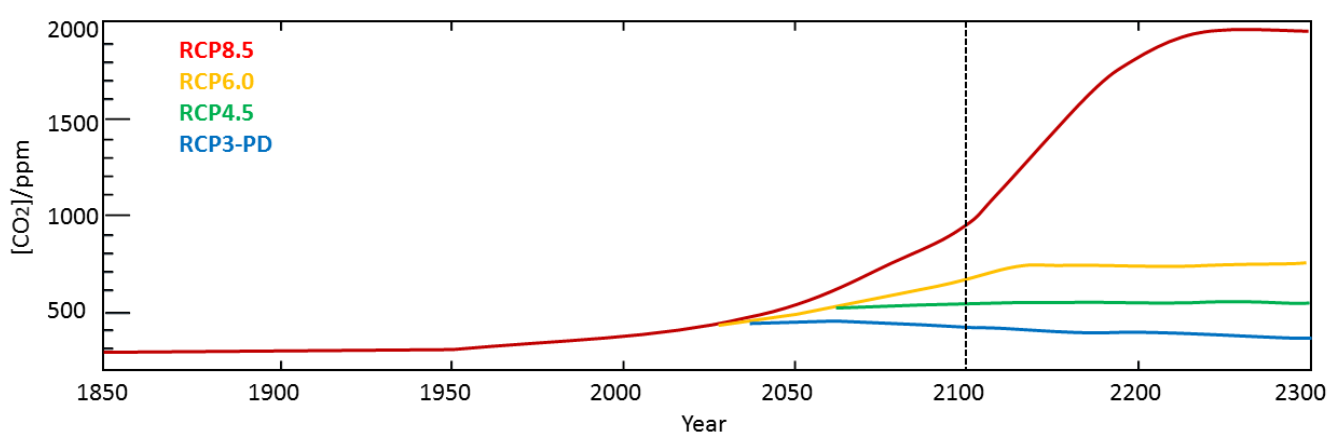

Figura 1 - Concentrações em ppm (parte por milhão) de CO2, incluindo a série Historical, que compreende o período entre 1860 até 2300.Fonte: Baseado em Jones et al. (2011). 
proposta, inicialmente, por Hoskins et al. (1985), sobre o uso dos mapas de vorticidade potencial. A análise por meio da vorticidade potencial permite descrever, em detalhes, os processos de formação e intensificação de fenômenos sinóticos, entre eles, os bloqueios. Hoskins definiu que valores absolutos de VP menores que 1,5 UVP indicam presença de ar troposférico, enquanto ar estratosférico tem valores absolutos maiores que 4,0 UVP. Para a tropopausa dinâmica é definido um valor absoluto de 2,0 UVP. Durante a análise, é importante notar que a VP é quase sempre positiva no Hemisfério Norte e negativa no Hemisfério Sul (exceto em altos níveis na região do equador). Por convenção, valores de VP maiores (em módulo) que -1,5 UVP no Hemisfério Sul são anomalias anticiclônicas, enquanto os menores são anomalias ciclônicas.

Na média, a temperatura potencial, tanto em baixos como em altos níveis, possui um gradiente meridional positivo (sul-norte). Desse modo, encontram-se temperaturas mais altas próximas ao equador, diminuindo, gradativamente, na direção dos polos. Quando uma situação de bloqueio é observada, o anticiclone fica estacionado por um tempo mais prolongado em uma determinada região. A circulação do próprio anticiclone faz com que o ar mais quente das regiões equatoriais migre para latitudes mais altas e vice-versa, como visto na figura 2. Assim, há uma quebra no gradiente meridional comumente observado. A persistência durante alguns dias dessa situação leva a concluir que o escoamento pode estar entrando em uma situação de bloqueio.

A partir dessa ideia, o índice B é definido como a média da temperatura potencial de duas áreas (norte e sul), de $15^{\circ}$ de latitude por $5^{\circ}$ de longitude cada, conforme o esquema ilustrado na figura 2 . A dimensão da longitude deve-se ao fato de o índice poder ser calculado na posição em que se achar necessário e a alteração desse valor não influenciar, significativamente, os resultados. Já a dimensão da porção latitudinal (que será de $30^{\circ}$ ) deve-se

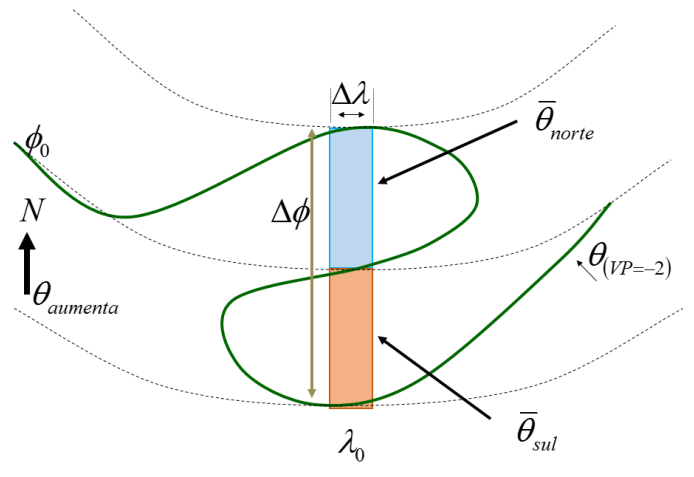

Figura 2 - Representação esquemática do cálculo do índice de bloqueio. As "caixas" possuem as dimensões de $5^{\circ}$ de longitude por $15^{\circ}$ de latitude, adaptado para o HS de Pelly e Hoskins (2003) ao fato de que a escala horizontal de abrangência dos bloqueios possui, aproximadamente, $3000 \mathrm{Km}$.

Desse modo, B, definido por Pelly e Hoskins (2003), é:

$$
B=\underbrace{\frac{2}{\Delta \phi} \int_{\phi_{0}}^{\phi_{0}+\frac{\Delta \phi}{2}} \theta d \phi}_{\text {setor } \text { norte }}-\underbrace{\frac{2}{\Delta \phi} \int_{\phi_{0}-\frac{\Delta \phi}{2}}^{\phi_{0}} \theta d \phi}_{\text {setor sul }}
$$

Sendo:

$\phi=$ latitude (em graus)

$\theta=$ temperatura potencial $(\mathrm{K})$.

$\lambda_{0}=$ longitude central (em graus).

Para o HS, quando o valor de é B > 0, o gradiente meridional de temperatura potencial na tropopausa dinâmica encontra-se em sua condição normal, nestas condições o fluxo médio de oeste é observado. Já quando $\mathrm{B}<0$, associado com uma persistência superior a três dias, poderá ser encontrada uma situação de bloqueio.

\section{Resultados e discussões}

Para verificar a funcionalidade do índice PH03, selecionou-se um evento de bloqueio atmosférico ocorrido na região do Pacífico sudeste. Esta região do globo concentra a maior parte das atividades de bloqueio que influenciam, de forma remota, os regimes de precipitação e temperatura na América do Sul (AS). Além desta, há duas outras regiões preferenciais de ocorrência no HS, concentrando-se próximo ao sudoeste da Austrália e na porção sudoeste do Atlântico, segundo Van LOON (1950).

Inicialmente, os dados do HadGEM2-ES foram pré -processados utilizando o modelo RegCM4, utilizando um espaçamento de grade de $150 \mathrm{~km}$, que, posteriormente, foram modificados para coordenadas isobáricas. Para o índice $\mathrm{PH} 03$, é necessário calcular a temperatura potencial $(\theta)$ em uma superfície de -2,0 UVP. Esta superfície encontra-se em altos níveis troposféricos (tropopausa) e possui certa variabilidade em relação à latitude. Além disso, essa variabilidade ocorre, em grande parte, devido à natureza dinâmica da atmosfera. Em casos de ciclogênese há um afundamento da tropopausa, já no estabelecimento de anomalias anticiclônicas ocorre um levantamento desta superfície.

O evento escolhido foi estudado por Marques e Rao (1999). O estudo analisa as diferenças entre bloqueios da região do Pacífico sudoeste em comparação aos que ocorrem na região da Nova Zelândia, além disso, discute 


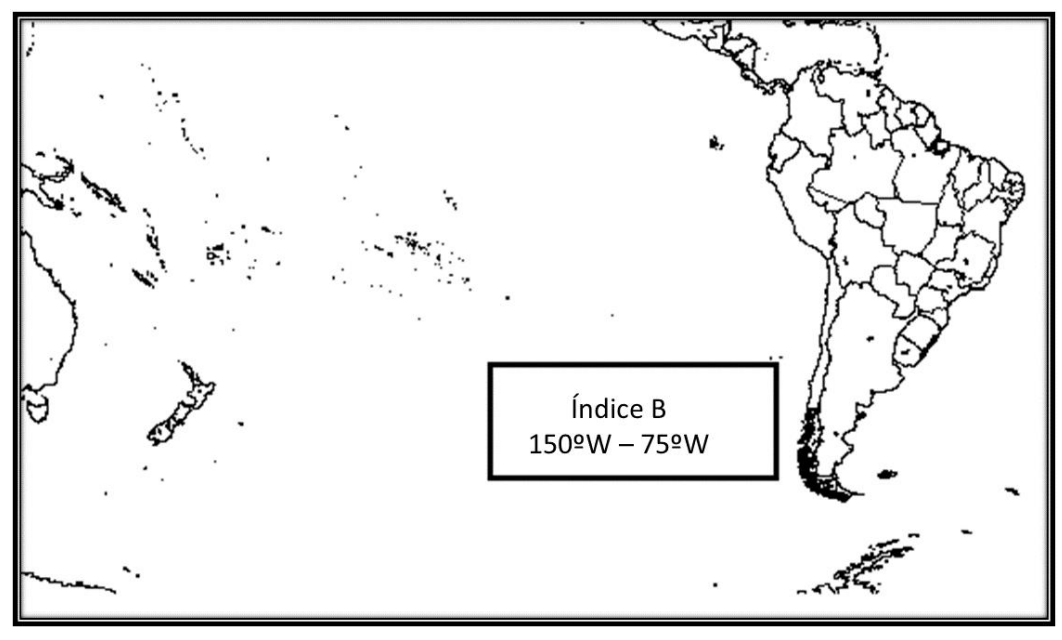

Figura 3 - Região do cálculo do índice de bloqueio PH03. A variação longitudinal está confinada entre 150॰ e75॰W. $\mathrm{O}$ cálculo foi repetido em intervalos fixos de $5^{\circ}$ de longitude

os efeitos dos bloqueios na circulação geral de inverno no HS e os efeitos na precipitação sobre a AS. No contexto deste trabalho, os efeitos remotos dos bloqueios serão analisados posteriormente, porém, nesta fase, a preocupação se concentra em verificar a funcionalidade do índice PH03. Deste modo, o índice será calculado para o período de inverno de 1986, além disso, será feita a análise dos campos meteorológicos que caracterizam o seu quadro sinótico. O bloqueio permaneceu ativo na região durante vários dias entre o final do mês de julho e a primeira quinzena de agosto.

Para a identificação da ocorrência do evento, o índice será calculado utilizando a latitude central $(\phi 0)$ fixa em $50 ॰ S$, sugerida por Berrisford et. al (2007). Os denominados "setor norte" e "setor sul" englobam todos os pontos de grade contidos dentro da "caixa" de 5॰ de longitude por $15^{\circ}$ de latitude, mostrado anteriormente na figura 2. A temperatura potencial média dentro de cada setor foi determinada pela função de média de área do GrADS. A região do pacífico estudada é mostrada na figura 3.

O resultado do cálculo é visto no diagrama de Hovmöller (longitude x tempo), na figura 4. Para ser considerado um bloqueio, o mesmo deve persistir por mais de três dias consecutivos e, ao mesmo tempo, estar presente em, no mínimo, duas faixas de longitude $\left(10^{\circ}\right)$. O fato de escolher a duração de três dias deve-se pela natureza mais transiente da circulação atmosférica no Hemisfério Sul. Além disso, mostra-se condizente com a análise de Berrisford et al. (2006), que utiliza a técnica do ajuste linear entre o número de eventos em escala logarítmica e a duração dos mesmos obtendo uma duração típica de 2,0 a 2,5 dias sobre latitudes mais altas.

Em Marques e Rao (1999), o período de ocorrência do bloqueio, identificado pelo índice de Lejeñas (1984), inicia no dia 29 de julho e se dissipa por volta de 14 de agosto de 1986, que foi corretamente captado pelo índice PH03. A figura 5a mostra os primeiros dias do mês de agosto, nos quais uma crista pronunciada toma forma próximo a $120 \circ \mathrm{W}$, advectando vorticidade anticiclônica a leste da mesma. Em superfície se observa o estabelecimento de um centro de alta pressão intenso (1050 hPa), que se mantém na região próxima a AS durante dias.

Pode-se observar, também, que o jato subtropical é desviado pelo sistema, como visto na figura $5 c$, passando ao sul do centro de alta pressão, que fica estacionado ali até o final da primeira quinzena de agosto. Como o HadGEM2-ES utiliza o calendário com ano composto por apenas 360 dias, não é possível ver se o modelo mostra de forma exata o dia e o horário de início e término do evento. Porém a identificação da condição de bloqueio pelo índice, a presença do sistema de alta pressão intenso localizado abaixo do cinturão das Altas Subtropicais estacionado durante dias, a presença vorticidade anticiclônica e o desvio da corrente de jato evidenciam, de forma clara, a ocorrência de um bloqueio. É possível ver, também, um centro de baixa pressão, presente no flanco equatorial do centro de alta pressão, classificando-o como um bloqueio do tipo dipolo.

\section{Conclusões}

Nesta pesquisa foram apresentados os resultados da fase de detecção de bloqueios atmosféricos na série de dados do modelo do Sistema Terrestre HadGEM2-ES. A importância de verificar se as simulações de longo prazo reproduzem, de maneira satisfatória, determinados sistemas meteorológicos é de suma importância, do ponto de vista que são estes sistemas que modulam o regime climático de cada região. Os bloqueios, apesar de não serem sistemas que atuam com grande frequência, em média 3 a 4 eventos por ano (Oliveira 2011), porém os mesmos atuam durante um período prolongado, fazendo com que as condições atmosféricas persistam durante sua atuação, aumentando, assim, o grau de acerto dos modelos. 


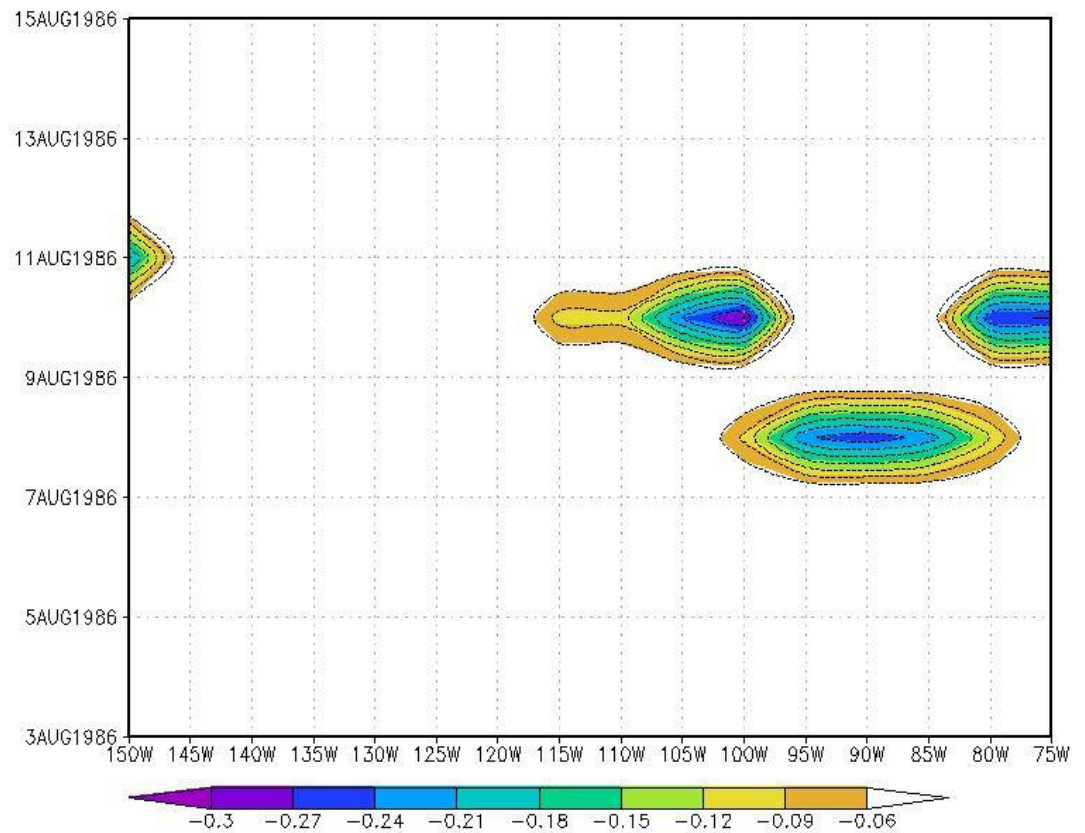

Figura 4 - Distribuição dos valores obtidos pelo índice PH03. Apenas o sinal negativo indica a presença de anomalias do gradiente meridional de $\theta$ forçado por quebras de onda de grande escala

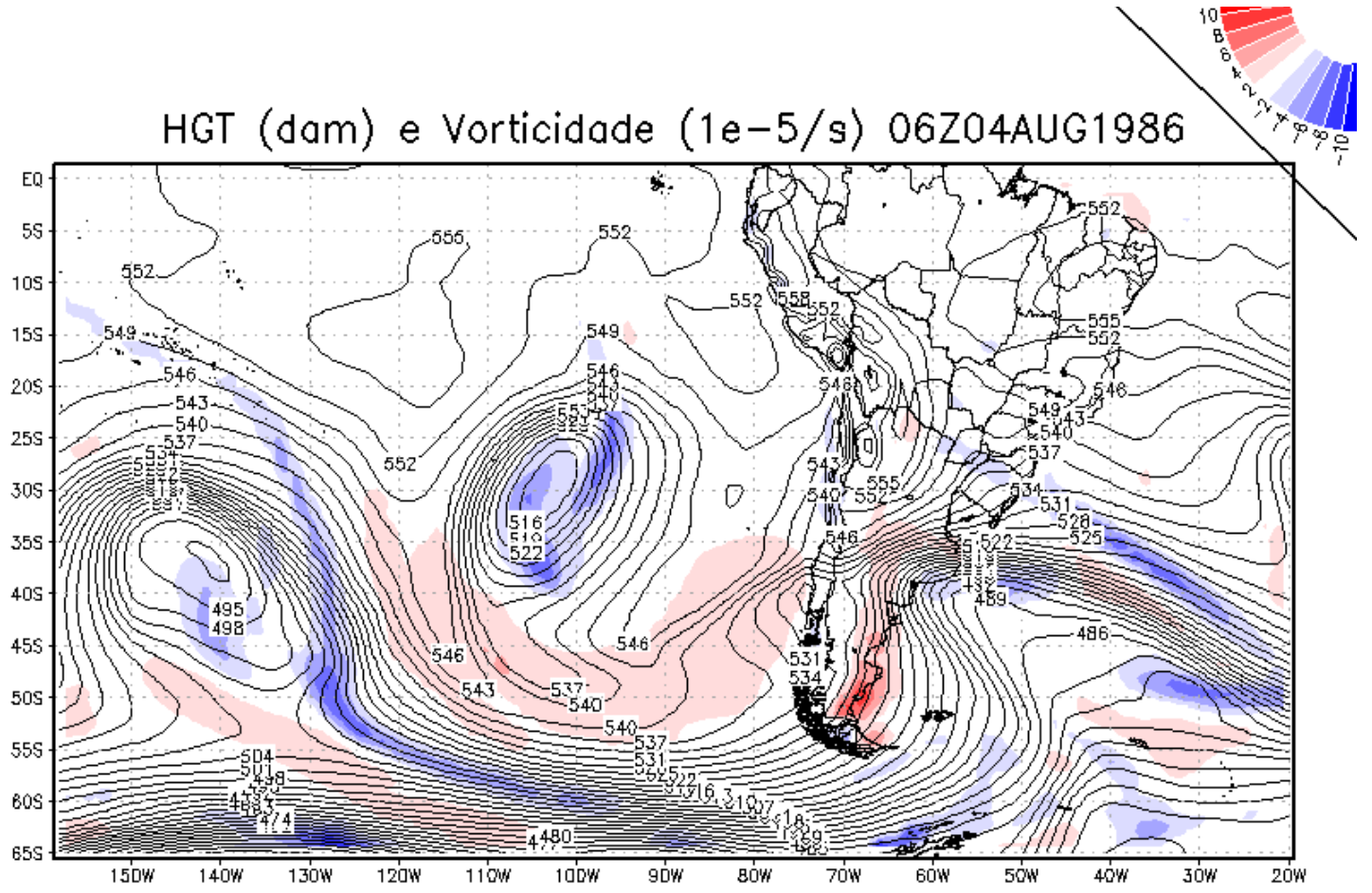

(a) Altura geopotencial (dam) e vorticidade $\left(1 e^{-5 s-1)}\right.$

Figura 5 - Campos meteorológicos indicando a configuração da situação de bloqueio atmosférico detectada pelo índice PH03

Continua... 


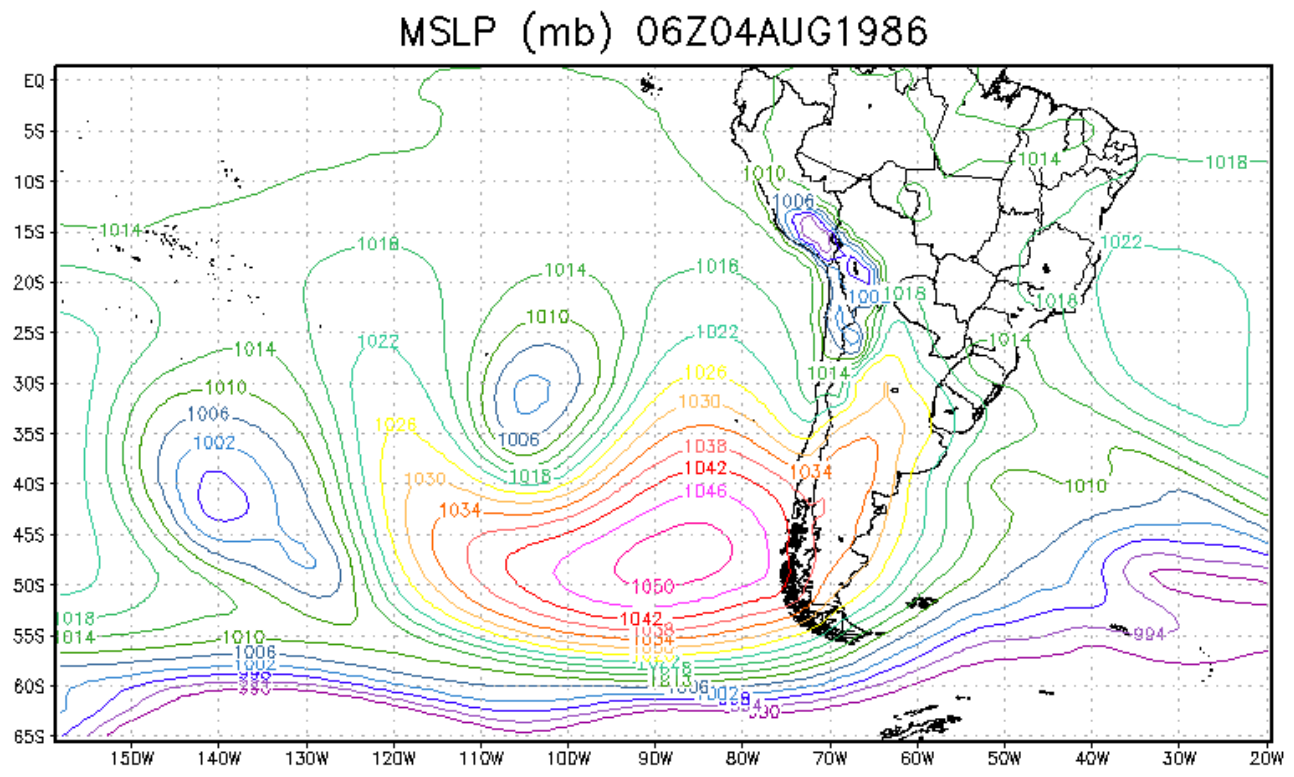

(b) Pressão reduzida ao nível do mar $(h P a)$

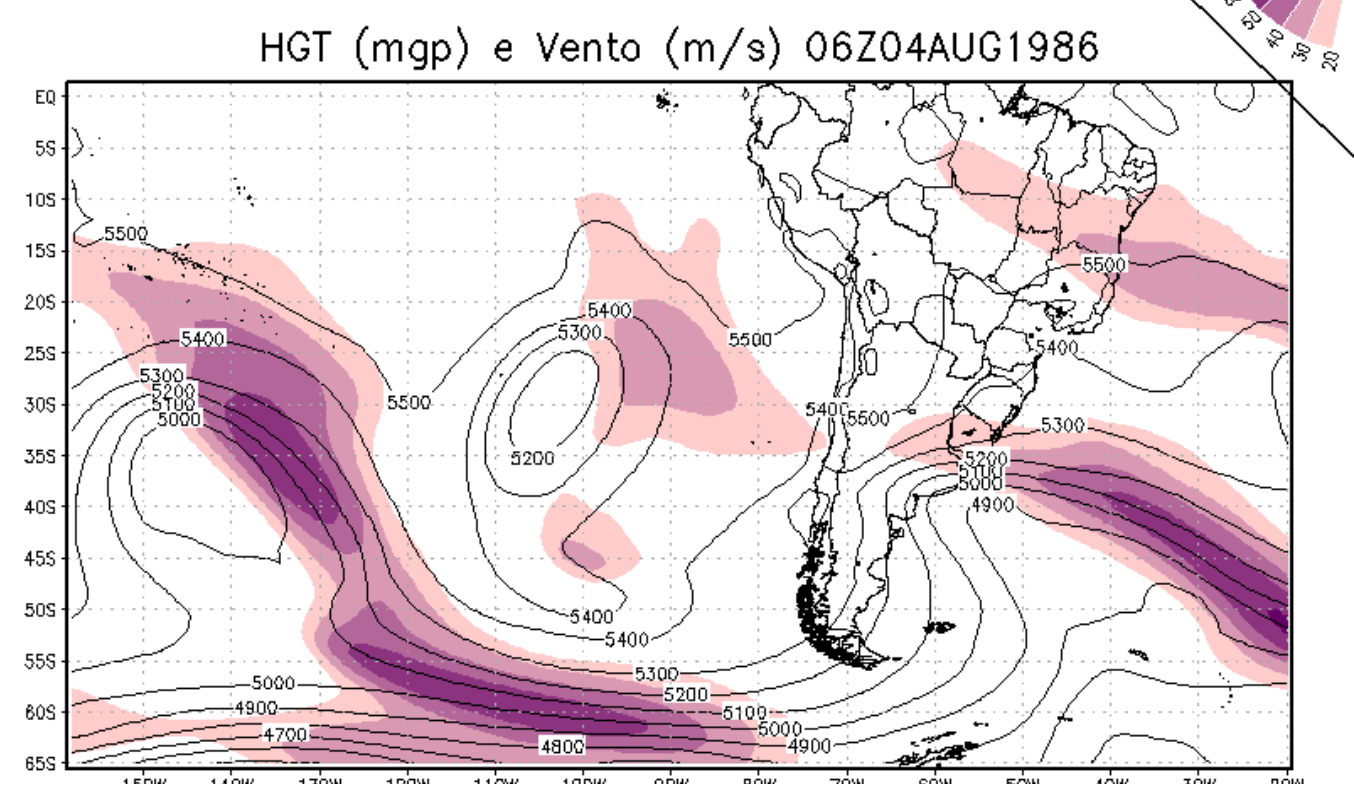

(c) Altura geopotencial em $500 \mathrm{hPa}(m g p)$ e vento em $250 \mathrm{hPa}(\mathrm{m} / \mathrm{s})$.

Figura 5 - Campos meteorológicos indicando a configuração da situação de bloqueio atmosférico detectada pelo índice $\mathrm{PH} 03$

O índice PH03 é o mais recente meio de detecção de bloqueios em campos atmosféricos, não existe, ainda, na literatura algum estudo sobre séries de tempo longas e de mudanças climáticas que utilizem este índice. Por se tratar de uma forma de caracterização mais dinâmica, deve-se fazer uma análise cuidadosa dos eventos que o índice considera um bloqueio de fato. No caso do evento de 1986, o índice se mostrou com boa acurácia, mostrando o sinal do evento no período correto, porém sua duração foi menor que aquela encontrada por Marques e Rao (1999). Como o propósito da pesquisa não é análise de caso, mas sim a alteração na frequência do regime de bloqueios em um cenário futuro, a detecção do sistema, em si, já é um resultado considerado satisfatório para compor a série de dados de clima futuro. 


\section{Agradecimentos}

Esta pesquisa é uma contribuição do Instituto Nacional de Ciência e Tecnologia (INCT- MC) para Mudanças Climáticas, financiado pelo projeto CNPq (Processo 573797/2008-0) e FAPESP (Fundação de Amparo à Pesquisa de São Paulo), (Processo 2008/57719-9) e da FA- PERGS (Fundação de Amparo à Pesquisa do Rio Grande do Sul).

\section{Referências}

BERRISFORD, P., HOSKINS, B. J., TYRLIS, E., 2006. Blocking and Rossby wave breaking on the dynamical tropopause in the Southern Hemisphere. Journal of Atmospheric Science 64, 2881-2898.

CAVALCANTI, I., FERREIRA, N., SILVA, M., SILVA-DIAS, M. (Eds.), 2009. Tempo e Clima no Brasil. Oficina de Textos.

COLLINS, W., , BELLOUIN, N., DOUTRIAUXBOUCHER, M., GEDNEY, N., 2008. Evaluation of the hadgem 2 model. Hadley Centre Technical Note (74).

FUJINO, J., NAIR, R., KAINUMA, M., MASUI, T., MATSUOKA, Y., 2006. Multigas mitigation analysis on stabilization scenarios using aim global model. Multigas mitigation and climate policy. J. Special Issue 27, 343-354.

HOSKINS, B. J., MCINTRYE, M. E., ROBERTSON, A. . W., 1985. On the use and significance of isentropic potential vorticity maps. Quarterly Journal of Royal Meteorological Society 111 (470), 887-946.

JONES, C. D., , HUGHES, J. K., BELLOUIN, N., HARDI- MAN, S. C., 2011. The hadgem2-es implementation of cmip5 centennial simulations. Geos- cientific Model Development 4 (3), 543-570.

LEJEÑAS, H., 1984. Characteristics of Southern Hemisphere blocking as determined from a time series of observational data. Quar- terly Journal of Royal Meteorological Soci- ety 110, 967-979.

MARQUES, R. F. C., RAO, V. B., 1999. A diagnosis of a long lasting blocking event over the Southeast Pacific Ocean. Monthly Weather Review 127, 1761-1776.

OLIVEIRA, F. N. M., 2011. Climatologia de Bloqueios Atmosféricos no Hemisfério Sul: observações, simulações do clima do século XX e cenários futuros de mudanças climáticas. Tese de Doutorado, Universidade de São Paulo, São Paulo.
PEIXOTO, J. P., OORT, A.H., 1992. Physics of climate. Springer.

PELLY, J., HOSKINS, B. J., 2003. A new perspective on blocking. Journal of the Atmospheric Sciences 60, 743-755.

REX, D. F., 1950a. Blocking action in the middle troposphere and its effect upon regional climate. Part I: an aerological study of blocking action. Tellus 2 (4), 196-211.

REX, D. F., 1950b. Blocking action in the middle troposphere and its effect upon regional climate. Part II: the Climatology of Blocking action. Tellus 2 (4), 196-211.

RIAHI, K., GRUEBLER, A., NAKICENOVIC, N., 2007. Scenarios of long-term socio-economic and environmental development under climate stabilization. Technological Forecaster Society 74 (7), 887-935.

VAN LOON, H., 1950. Blocking action in the middle troposphere and its effect upon regional climate. Part II: the Climatology of Blocking action. Tellus 2 (4), 196-211.

VAN VUUREN, D. P., DEN ELZEN, M. G. J., LUCAS, P. L., EICKHOUT, B., STRENGERS, B. J., VAN RUIJVEN, B., WONINK, S., VAN HOUDT, R., 2007. Stabilizing greenhouse gas concentrations at low levels: an assessment of reduction strategies and costs. Climatic Change 81 (2), 119-159.

WILLET, H. C., 1949. Long period fluctuations of the general circulation of the Atmosphere. Journal of Meteorology 6, 34-50.

WISE, M., CALVIN, K., THOMSON, A., CLARKE, L., BOND-LAMBERTY, B., SANDS, R., SMITH, S., JANE-TOS, A., EDMONDS, J., 2009. The implications of limiting $\mathrm{CO} 2$ concentrations for agriculture, land use, land-use change emissions and bioenergy. Nortwest Pacific National Laboratory - Technical Note PNNL17943, 1-40. 